\title{
A GEOGRAFIA DA SAÚDE NO ENSINO FUNDAMENTAL
}

\section{ARTIGO DE REVISÃO}

NASCIMENTO, Márcio Moreira Do ${ }^{1}$

NASCIMENTO, Márcio Moreira Do. A Geografia da Saúde no Ensino Fundamental. Revista Científica Multidisciplinar Núcleo do Conhecimento. Ano 05, Ed. 06, Vol. 06, pp. 86-95. Junho de 2020. ISSN: 2448-0959, Link de acesso: https://www.nucleodoconhecimento.com.br/educacao/a-geografia

\section{RESUMO}

O presente artigo tem por finalidade o estudo e a compreensão da Geografia da Saúde e sua utilização no ensino fundamental. A Geografia da Saúde possui estratégias de estudo para as distribuições na assistência médica de forma macro e microrregional, bem como a previsão e relatórios sobre as estatísticas de epidemias e outros problemas de saúde. Por essa grande responsabilidade é que os estudos sobre as políticas de ensino da Geografia da Saúde devem acontecer na educação. Os temas ligados a Geografia da Saúde devem ser trabalhados e ou adaptados pelos docentes para a conscientização e prevenção sobre os principais problemas de saúde. Foi usado o método de revisão bibliográfica para a realização do estudo e produção do artigo, a pesquisa apontou para uma real necessidade de mais estudos e divulgação de temas relacionados à Geografia da Saúde.

Palavras-Chave: Geografia da Saúde, ensino de Geografia, desafios na Geografia.

\section{INTRODUÇÃO}

Os aspectos ambientais são os principais agentes dos males de saúde que afetam a população. Com a acelerada degradação do meio ambiente impactos afetam

\footnotetext{
${ }^{1}$ Mestrando em Geografia (UFMS-Aquidauana). Especialização em Gestão Escolar. Graduação em Pedagogia.
} 
diretamente o próprio homem nas questões da saúde. A Geografia da Saúde exerce fundamental papel para que sejam explicados esses fenômenos e amenizadas as consequências.

Para a compreensão da importância do estudo da Geografia da Saúde e o ensino de sua disciplina, foi feita uma revisão bibliográfica e documental buscando explanar um breve histórico do que se trata o objeto de nosso estudo traçando as respostas pelos questionamentos presentes no artigo.

O objetivo principal é compreender os métodos e funções a encargo da Geografia da Saúde e as didáticas da disciplina aplicadas à educação básica. Com a pesquisa foi possível a compreensão dos principais desafios enfrentados por esse ramo da Geografia que busca mapear e estudar a proliferação de algumas doenças, bem como a determinação do processo de sua causa, a importância do ensino da Geografia da Saúde diante da globalização, onde se faz necessário a articulação dos saberes para que se chegue a compreensão de determinados processos.

\section{GEOGRAFIA DA SAÚDE - ASPECTOS GERAIS}

A Geografia enquanto estudo do espaço, aborda múltiplas faces em sua aplicação de pesquisa, uma delas é a saúde, analisando as epidemias, o sanitarismo, determinando os fatores sociais das doenças, os complexos patogênicos propostos por Sorre (1955) que compreendiam as relações entre as doenças e o espaço geográfico. Para Guimarães (2015) a saúde é objeto de estudo de geógrafos pela ligação direta com o desenvolvimento das cidades, apesar de que enquanto a Epidemiologia faz uso constante das ferramentas da Geografia, o contrário ocorre com menos frequência.

Um importante estudo realizado pelo geografo brasileiro Josué de Castro, revelou a importância da atuação da Geografia da Saúde e sua metodologia para o levantamento de dados. Castro (1984) diz que sua obra é um estudo de natureza ecológica sobre a fome no Brasil, baseado nos princípios da localização, causalidade, extensão, unidade terrestre e correlação. 
O estudo de Josué de Castro revelou dados da subnutrição e fome crônica nas regiões brasileiras, identificando, a causa para diversas doenças e males sociais, ocasionando a evasão escolar.

Outro conceituado geografo brasileiro que se dedicou a disseminar a importância dos estudos da Geografia aplicados na saúde foi Milton Santos. Para Faria e Bortolozzi (2009) Santos relata que as condições espaciais da propagação de esquistossomose revelou que foi a evolução urbana que propagou a doença, pois através da expansão urbana do local afetado, a doença encontrou as condições ecológicas para a sua propagação, esse estudo revelou um avanço no descobrimento das causas de proliferação de doenças contagiosas, pois trouxe as questões ambientais, sendo antes avaliadas como causas das imigrações, agora pela globalização e a facilidade de mobilidade da população propiciam o avanço das epidemias em áreas urbanas de forma muito mais rápida.

O território deve ser o campo para as análises da Geografia em Saúde, e levar em conta o e as práticas de saúde analisadas. Como enfatiza Bortolozzi e Faria:

[...] duas questões se colocam fundamentais para uma prática territorial em saúde. Primeiramente, deve-se ter claro o que se entende pela categoria território, uma vez que é essa definição que vai fornecer os parâmetros para a identificação de áreas para a implementação de ações práticas em saúde. Em segundo lugar, deve-se entender quando e quais as práticas de saúde podem ser compreendidas numa análise territorial (2009, p.36).

Milton Santos relata o território como apropriação social, sendo assim as permissões de recortes territoriais foram ampliadas, por ser o território algo dinâmico, passível de transformações, as diversas funções e usos que o território apresenta segundo o jogo de interesses entre poder e a sociedade é que determina as ações aplicadas para a saúde (SANTOS, 1988). 
A relação dialética do território com as interações locais e globais permite que as ações sociais como saúde, educação aconteçam, sendo assim a eficácia dos serviços de saúde pública dependem de uma boa gestão e estudo territorial.

A relação de saúde e conceitos geográficos não é nenhuma novidade:

O paradigma hipocrático, geralmente considerado a matriz do pensamento médico do mundo ocidental, já considerava o ambiente das cidades como um foco de agravos à saúde. Se as doenças eram compreendidas como o desequilíbrio de diferentes fluidos (sangue, água, bílis e fleuma), por sua vez, a saúde era vista como o resultado do equilíbrio entre esses fluidos em razão das condições ambientais dos lugares (GRMEK, 1999 apud GUIMARÃES, 2015, p.18).

A urbanização do mundo industrial e a falta de saneamento adequado provocou de forma rápida a proliferação de doenças contagiosas, sendo o saneamento básico o único remédio para conter as doenças durante muito tempo, esse período ficou conhecido como Sanitarismo, "de 1830 e 1875, a saúde pública e o planejamento urbano foram considerados uma mesma entidade" (GUIMARÃES, 2015, p.24).

A prática sanitária oferece as bases da epidemiologia e da geografia como conhecimentos que irão trabalhar, mesmo que de forma distinta, o conceito de espaço para os estudos ligados à saúde (BARCELLOS et al, 2008).

Os estudos geográficos se fazem necessários no campo da saúde, para se buscar soluções para enfrentamento dos problemas da urbanização oriundos da industrialização e da globalização os estudos da microbiologia permitiram que se definissem programas de controle ambiental e combate a diversas patologias infectocontagiosas.

É no interesse dos lugares como interação social entre pessoas e o meio, buscou-se a explicação de como os lugares impactam na saúde. Dessa forma, busca-se a associação de como as ações humanas em determinado meio influenciam na promoção e prevenção de doenças (ALIEVE; PINESE, 2014). 


\subsection{PRINCIPAIS CONCEITOS DA GEOGRAFIA DA SAÚDE}

O maior desafio do geografo que se propõe a estudar as questões da saúde é que seu estudo afeta diretamente a vida das pessoas, logo, não deve ser passível de erros. "O desafio é pensar a saúde a partir do espaço, discutindo os problemas teóricos que envolvem o olhar dos geógrafos do ponto de vista dos sujeitos sociais" (GUIMARÃES, 2015, p.41).

Sendo ligada estreitamente ao bem-estar e qualidade de vida, a saúde deixou de ser campo de estudo biológico para integrar o quadro sociocultural. A Organização Mundial da Saúde define doença como uma má adaptação do organismo aos fatores ambientais e disfuncionalidades do organismo e saúde como um bem-estar físico, mental e social (SANTANA, 2014).

Sendo a Geografia da Saúde a ciência que abrange aspectos da Geografia Física e Humana, intercruza informações entre fenômenos naturais, socioculturais e comportamentais na explicação para a saúde e doença.

O objetivo da Geografia da Saúde e dos cuidados de saúde é relativo ao estudo geográfico da distribuição e acesso dos serviços de saúde e a avaliação das desigualdades em saúde. Têm vindo a ser privilegiados alguns temas como a organização, acesso e utilização dos cuidados de saúde e, mais recentemente, a otimização da localização de equipamentos de saúde e planificação de serviços de saúde (SANTANA, 2014, p.19).

Para a realização dos estudos são utilizados métodos como: a construção de relatórios matemáticos para definir a eficiência e acessibilidade dos serviços de saúde com base nos dados dos doentes; cálculo de distância dos serviços de atendimento; estudos de Medicometria regional; estudos sobre a dinâmica do comportamento do paciente. 
Os conceitos que fazem parte do estudo da Geografia da saúde são: a epidemiologia que é o estudo das doenças e os fatores da propagação na população, a tríade dos fatores ambientais que podem ser classificados em biológicos, sociais e físicos; a tríade do modelo ecológico que se define em: agente, hospedeiro e ambiente, onde o fluxo desses fatores através na dinâmica de suas transformações podem alterar um quadro de doença, propaga-la ou erradica-la (BARATA; BRICEÑO-LEÓN, 2000).

Para Guimarães (2015) os estudos sobre a saúde requerem a compreensão de que o corpo humano não é apenas anatomofisiológico, mas também social.

Para tanto é necessário lançar mão de conceitos técnicos, desenvolvidos por Milton Santos para caracterizar o espaço estudo da saúde, com Sistemas técnicos e inovação tecnológica; - Periodização e meio geográfico; - Exclusão social e pobreza urbana (GUIMARÃES, 2015, p.59).

Sendo assim esses conceitos aplicados ajudam o geografo nos estudos e compreensão da saúde e o incumbe na tarefa da análise da dimensão social das doenças para o enfrentamento de surtos epidemiológicos e auxiliar nos problemas de saúde apresentando entre a população.

\section{A GEOGRAFIA DA SAÚDE NO ENSINO BÁSICO}

Os Parâmetros Nacionais da Educação (PCN) de 1998, abordam a saúde como tema transversal referente a uma abordagem social. Dessa forma está vinculada à todas as disciplinas em sua abordagem. Falar em saúde é tratar de forma ampla por exemplo da qualidade do ar que se respira, a miséria, fragilidade social e a desnutrição, e inserção das da população no mundo do trabalho, a forma de vida pessoal (BRASIL, 1998).

Pela abordagem do MEC para a temática da saúde percebeu-se que a Geografia tem função fundamental para a aplicação dos conceitos sobre o espaço, as interações sociais e sua relação direta com a saúde e doença. Para Braga (2013) na escola o 
conteúdo se oculta no currículo e muitos professores não conhecem esta subdivisão da Geografia.

O ensino da Geografia no ensino fundamental tem como objetivo em identidade sociocultural estabelecer a conexão das transformações e usos dos lugares e analisar a transformações feitas pelas sociedades; para Transformações das paisagens naturais e antrópicas, a identificação da transformação da paisagem e relações com o surgimento das cidades; em biodiversidade e ciclo hidrológico, uma análise da relação homem-natureza em dimensão social, no que afeta as transformações da biodiversidade local e mundial; e em atividades humanas e dinâmica climática analisar as consequências da ação humana influenciando no clima (BRASIL, 1998). Esse exemplo apenas ilustra a importância e a abrangência que as questões estudadas em Geografia refletem nos estudos também relacionados à saúde.

Como foi visto no decorrer do artigo, as ações do homem, a industrialização, as transformações climáticas exercem influência direta nas epidemias e doenças infectocontagiosas, dessa forma a Geografia direcionada para o estudo dos fenômenos da saúde como forma de identificação e prevenção dos fatores que causam os malefícios na saúde populacional.

Para Braga (2017) por mais que nos livros didáticos encontramos conceitos da Geografia da Saúde, os professores não abordam o tema em sala de aula. O autor propõe que desde a sétima série o professor peça que os alunos escolham uma Unidade Básica de Saúde e a partir de sua escolha tenham dados sobre a densidade demográfica, dados sobre saneamento básico e riscos socioambientais.

O objetivo da pesquisa é fazer com que o aluno entenda que a localização geográfica de uma Unidade Básica de Saúde não se dá de qualquer forma e sim conforme a carência de Saúde Pública da população. Por esse motivo, bem provável que uma população numerosa, sem acesso a saneamento básico e que apresenta um risco ou vulnerabilidade socioambiental, estará mais exposta a determinados 
agentes infecciosos que podem desencadear doenças inerentes à insalubridade $(2017$, p. 17.505).

Os alunos precisam conhecer e se adaptar aos termos aplicados à Geografia da saúde, como pandemia, epidemia Braga (2017) sugere que os conceitos e doenças devam ser trabalhados juntos à Geografia e População, isso pode ser feito através da confecção de mapas com a expectativa de vida da população local, relacionando conceitos de espaço e modificações climáticas e ambientais.

Todas as perspectivas ensaiadas para o ensino fundamental parte de conceitos de Geografia já aplicados em sala de aula, porém que devem ser adaptados para inserir esses novos conhecimentos que são tão úteis para o exercício da cidadania no combate preventivo de doenças.

A Geografia é responsável por desenvolver ações de reflexão para o espaço onde se vive e também o estudo da Geografia visa o conhecimento do lugar onde se vive para a melhor preservação e qualidade de vida, a Geografia da Saúde abordada em ambiente escolar deve se focar nas principais questões locais ou de preocupação geral (como epidemias de sarampo, febre-amarela para a plena conscientização dos alunos e ação nas Unidades Básicas de Saúde.

Para Braga (2015) através dos parâmetros curriculares da SEED, é necessária a substituição da Geografia tradicional pela Geografia Crítica como disciplina aplicada ao Ensino Médio nas Redes Estaduais de Ensino. Essa reestruturação permite o diálogo com os conteúdos transversais previstos na BNCC, que são: ética, cidadania, pluralidade cultural, saúde, orientação sexual e temas locais (BRASIL, 1998).

Para Paula et al (2018) a Geografia da Saúde nos estudos das populações representa não apenas a quantificação dos dados coletados, mas também a qualificação na interpretação e entendimento sobre o objeto estudado. Para que o professor se adapte a abordagem da Geografia da Saúde nas aulas é necessário que recorra a uma pedagogia de atuação construtivista, onde a sua atuação em aula passe de transmissor do conhecimento para mediador do mesmo. 
Um exemplo de ação escolar voltada para a Geografia da Saúde que beneficia a população a partir da escola, é a prevenção e combate à dengue. Os alunos devem ser instruídos para entender como funcionam as doenças que se tornam epidemias e as medidas para que se evite o contágio, as medidas preventivas devem ser especificadas e repetidas para toda a comunidade escolar.

Um estudo realizado por Paula et al (2018) com alunos que estudaram os conceitos da Geografia da Saúde em um projeto sobre a dengue revelou que a partir das discussões inseridas com o tema, foi apresentado um resultado que vai além dos livros didáticos. Os alunos apresentaram uma postura de comprometimento com a responsabilidade em cuidados ambientais para as ações de combate aos criadouros dos vetores, trabalhando com a conscientização dos alunos a sociedade inteira sai ganhando.

\section{CONCLUSÃO}

O artigo apresentado revelou os aspectos que compõe o estudo da Geografia da Saúde, como a utilização do conceito de espaço e paisagem para determinar fatores correspondentes a dados da saúde de determinada região. A contribuição de grandes geógrafos brasileiros, como: Josué Castro e Milton Santos, trouxeram grandes reflexões para a ciência, porém ainda no Brasil existem poucos estudos relacionados a temática da saúde.

A Geografia da Saúde está ligada à vida humana, a qualidade de vida, a prevenção e combate a doenças infectocontagiosas e às melhorias na acessibilidade do serviço público de atendimento, portanto é necessário que mais estudos sejam desenvolvidos a fim de divulgar essa área de atuação da Geografia.

Nas escolas, tanto no ensino fundamental como no ensino médio, a saúde entra como conteúdo transversal que deve ser aplicado aos alunos junto aos componentes básicos da disciplina. 
Nos livros didáticos e nas orientações do Ministério da Educação, a Geografia da Saúde aparece em diversos pontos de ligação com o estudo da Geografia tradicional e crítica, cabe aos docentes trabalharem os pontos de conexão para que os alunos entrem em contato com a realidade de seu bairro a partir do estudo das Unidades Básicas de Saúde.

A temática Geografia da População é fundamental para a introdução de conceitos de pesquisa da saúde por já conter em sua diretriz um estudo que conduz a caracterização da saúde como decorrência dos fatores sociais, políticos e econômicos do lugar. Sendo assim, o aluno será incorporado ao estudo de questões atuais da saúde, de preferência que afete a sua região para criar as noções necessárias ao desenvolvimento desse campo da ciência.

Geografia da Saúde constitui importante ferramenta na gestão das populações e controle de doenças, bem como na organização da implantação e melhora na acessibilidade dos serviços de saúde. Para a educação escolar, deve constar em forma de atividades que promovam a noção de preservação ambiental e combate aos malefícios que afligem a população. Sendo conscientizados das ações preventivas que a Geografia da Saúde preconiza para as epidemias, tomando medidas que serão amplamente disseminadas no convívio social e na comunidade escolar.

\section{REFERÊNCIAS}

ALIEVE, Alan Alves; PINESE, José Paulo de. A geografia da saúde no Brasil: precedentes históricos e contribuições teóricas. Paraná: Universidade Estadual de Londrina, [2014]. Disponível em: $<\mathrm{http}$ ://observatoriogeograficoamericalatina.org.mx/egal14/Geografiasocioeconomica /Geografiamedica/01.pdf> Acesso em: 20/08/2019.

BARATA, Rita Barradas; BRICEÑO-LEÓN, Roberto (orgs.). Doenças endêmicas: abordagens socias, culturais e comportamentais. Rio de Janeiro: FioCruz, 2000. 
BARCELLOS, Christovam, et al., orgs. Território, ambiente e saúde. Rio de Janeiro: FioCruz, 2008.

BORTOLOZZI, Arlêude; FARIA, Rivaldo Mauro de. Espaço, território e saúde: contribuições de Milton Santos para o tema da geografia da saúde no Brasil. In: R. RA'E GA, Curitiba, n. 17, p. 31-41, 2009. Editora UFPR.

BRAGA, Ramon de Oliveira Bieco. A Geografia da Saúde na Geografia Escolar do Ensino Médio, no contexto dos Colégios Estaduais de Curitiba/PR: uma análise crítica. Curitiba: Universidade Federal do Paraná, 2015. Disponível em: <https://acervodigital.ufpr.br/bitstream/handle/1884/39889/R\%20-\%20D\%20\%20RAMON\%20DE\%20OLIVEIRA\%20BIECO\%20BRAGA.pdf?sequence=1\&isAllo wed $=\mathrm{y}>$ Acesso em: 20/08/2019.

BRAGA, Ramon de Oliveira Bieco. Por uma Geografia da Saúde no Ensino Fundamental. In: XI Congresso Nacional de Educação, Paraná, 2013. P. 17.50017.511

BRASIL. (Ministério da Educação e Cultura). Base Nacional Comum Curricular. Educação Infantil e Ensino Fundamental. 2017. Disponível em: http://basenacionalcomum.mec.gov.br/wp-content/uploads/2018/02/bncc20dezsite.pdf. Acesso em: 21/08/2019.

BRASIL. Secretaria de Educação Fundamental. Parâmetros curriculares nacionais: terceiro e quarto ciclo do ensino fundamental - introdução aos parâmetros curriculares nacionais. Brasília: MEC/SEF, 1998.

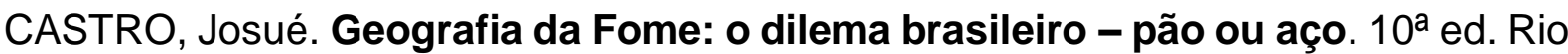
de Janeiro: Edições Antares, 1984.

GUIMARÃES, Raul Borges. Saúde: Fundamentos da Geografia Humana. São Paulo: Editora Unesp, 2015. 
PAULA. Hugo Ribeiro Borges de., et al. Por uma abordagem da Geografia da Saúde no Estudo da População: A espacialização do caso da dengue em Londrina/PR. Fortaleza: GeoSaberes, $2010 . \quad$ Disponível em: <file://C:/Users/Lenovo\%20i3/Downloads/623-1-2839-1-10-20171228\%20(3).pdf>. Acesso em: 20/08/2019.

SANTANA, Paula. Introdução à Geografia da Saúde: território, saúde e bem-estar. Coimbra: Imprensa da Universidade de Coimbra, 2014.

SANTOS, Milton. Metamorfoses do Espaço Habitado: fundamentos teóricos e metodológicos da geografia. São Paulo: Hucitec, 1988.

SORRE, M. Fundamentos biológicos de la Geografia humana. Barcelona: Editorial Juventud, 1955.

SORRE, M. El hombre en la Tierra. Barcelona: Labor, 1967.

Enviado: Maio, 2020.

Aprovado: Junho, 2020. 